\title{
Tungiasis afecta a un turista chileno
}

Cristian Vergara M., Elizabeth Barthel M., Eduardo Labarca M., Patricia Neira O. y Roberto Espinoza E.

\section{Tungiasis affects a chilean turist}

Tungiasis is a cutaneous ectoparasitosis caused by the female sand flea Tunga penetrans whose higher prevalence occurs in Sub-Saharan Africa, South America and the Caribbean. We report a case of a 23 year old chilean male who presented dermal lesions suggestive of tungiasis on his return from Brazil. The diagnosis was confirmed by biopsy, identifying the arthropod and an egg from one of the lesions. The natural history, co-morbidities and treatment options were reviewed.

Key words: Tungiasis, Tunga penetrans, ectoparasitosis, parasites, sand flies.

Palabras clave: Tungiasis, Tunga penetrans, ectoparasitosis, parasitos, mosca de arena.

\section{Introducción}

$\mathrm{L}$ a tungiasis es una ectoparasitosis cutánea causada por la penetración de la pulga hematófaga Tunga penetrans (Phylum Arthropoda, Clase Insecta, Orden Siphonaptera, Familia Tungidae) en la epidermis de un hospedero; conocida comúnmente como "pulga de arena", o "bicho do pé", "bicho porco" (Brasil), "chique", "nigua" (Paraguay, México), "pique" (Argentina, Uruguay), "chica" (Colombia), "pico" (Perú), "suthi pique" (Bolivia) y en países americanos de habla inglesa "sand flea", "jigger flea", "chigoe", "borrowing flea".

La infestación por $T$. penetrans se caracteriza por la formación de lesiones papulares en la piel, con un halo eritematoso y luego blanquecino, con un punto central pardo negruzco que corresponde a la zona posterior del abdomen de la pulga. Las áreas de la piel más afectadas son los ortejos, región peri-ungueal, plantar y los talones ${ }^{2,3}$; sin embargo, la infestación se ha detectado también en otros sitios ectópicos, incluyendo las manos, codos, muslos, la región glútea ${ }^{4}$ y los párpados superiores 5 .

La pulga se distribuye principalmente en áreas de África Sub sahariana, Sudamérica y el Caribe. En estas áreas constituye una infestación endémica y está relacionada a condiciones de pobreza ${ }^{2,3,6}$. En otras áreas del mundo el reporte de casos corresponde a turistas afectados que visitaron las áreas de endemia ${ }^{7-9}$.

Se comunica el caso clínico de un joven chileno que viajó a Brasil a una zona de alta prevalencia de tungiasis, infestándose por $T$. penetrans. Creemos que el escaso conocimiento en nuestro medio de sus manifestaciones clínicas amerita su comunicación, para ser reconocido oportunamente.

\section{Caso Clínico}

Varón de 23 años, estudiante universitario, sin antecedentes mórbidos, que viajó a Brasil junto a 50 compañeros por motivos académicos, permaneciendo dos semanas en Cariaba, pequeña localidad costera al sur de Porto Seguro, en el estado de Bahía. En ese lugar, el grupo permaneció gran parte del tiempo en la playa a pies descalzos y se bañaban en la desembocadura de un río. Una semana después de su regreso a Chile, presentó lesiones cutáneas tipo pápulas pardo negruzcas, pruriginosas, de 2 a $3 \mathrm{~mm}$ de diámetro, ubicadas en las zonas peri-ungueales de los ortejos y plantas de ambos pies (Figuras 1 y 2), rodeadas de un halo eritematoso y posteriormente dolorosas. Tanto él como la mayoría del grupo presentaron lesiones de similares características y localización. Motu proprio el paciente decidió consultar a la policlínica de Infectología del hospital.

Considerando las características de las lesiones, los antecedentes epidemiológicos del área visitada y una revisión bibliográfica, se planteó el diagnóstico de tungiasis, indicándose la extirpación quirúrgica. Previo a la cirugía, el paciente manipuló las lesiones entregando al médico tratante el material extraído.

El tratamiento consistió en la extirpación y curetaje del lecho de las lesiones, no presentando complicaciones locales. El estudio histológico de las lesiones extirpadas mostró cambios compatibles con tungiasis (Figura 3). El material extraído por el paciente fue analizado en el laboratorio de parasitología de la Universidad de Valparaíso, lugar donde se logró identificar parte del abdomen del artrópodo y apéndices locomotores (Figura 4 y 5) e identificación de un huevo (Figura 6). Se indicó profilaxis antitetánica con toxoide y no se utilizó antibacterianos.
Facultad de Medicina. Universidad de Valparaíso, Chile. Escuela de Medicina Dirección de Post Grado y Post Título Departamento de Medicina Interna (CVM)

Cátedra de Parasitología (PNO) Hospital Naval Almirante Nef, Viña del Mar, Chile Servicio de Medicina (EBM) Servicio de Cirugía (ELM) Servicio de Anatomía Patológica (REE)

Recibido: 12 de mayo 2008 Aceptado: 23 de octubre 2008

Correspondencia a: Cristián Vergara Melian vergaravalpo@gmail.com 


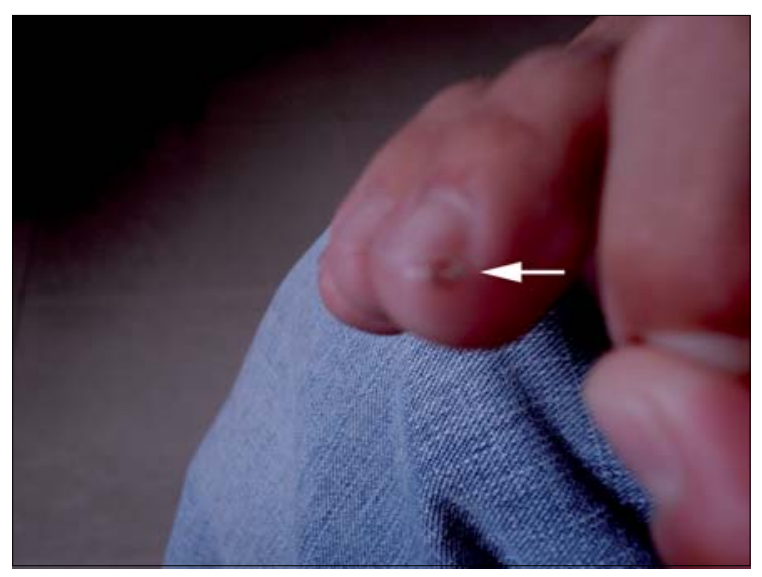

Figura 1. Lesión cutánea en la zona peri-ungueal de ortejo del pie derecho (Flecha).

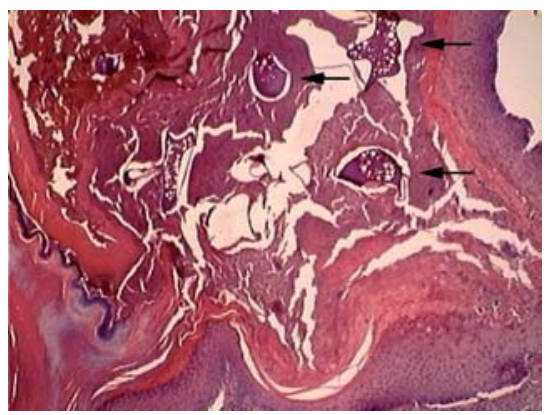

Figura 3. Fotografía de la piel (hematoxilina-eosina 10x). Se muestran los huevos del parásito en forma de cuerpos basófilos (flecha) en el estrato córneo.

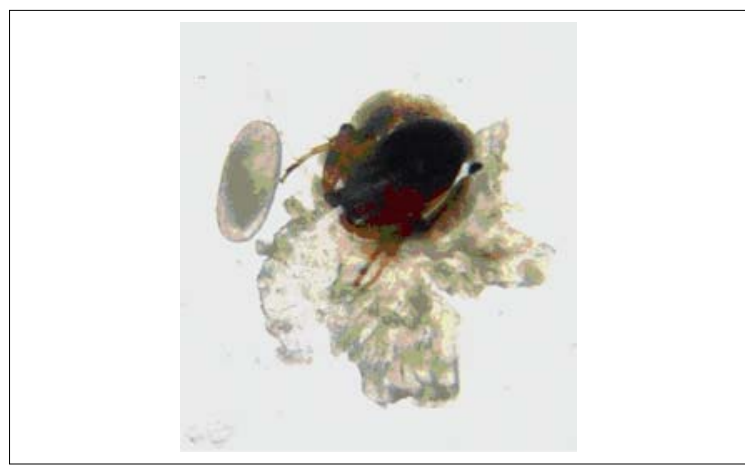

Figura 5. Visión dorsal de hembra de T. penetrans extraída por e paciente de la lesión y huevo obtenido por manipulación microscópica (Aumento 10x).

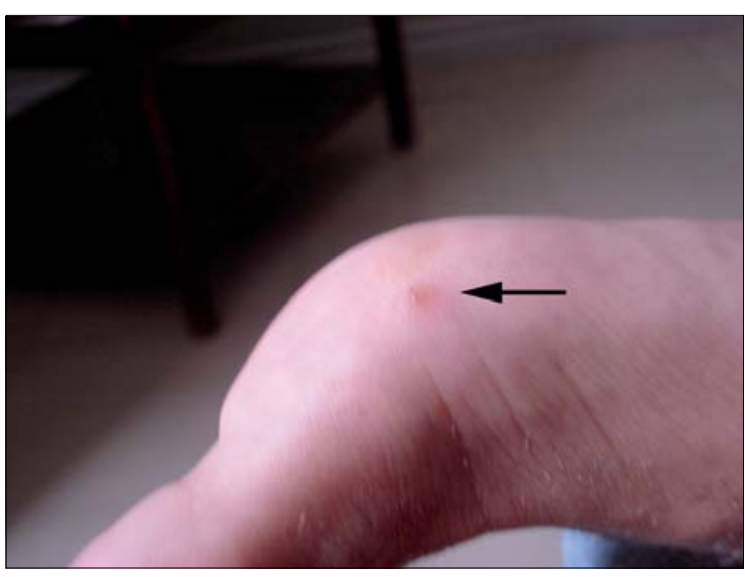

Figura 2. Lesión cutánea plantar del pie izquierdo manipulada por el paciente (Flecha).

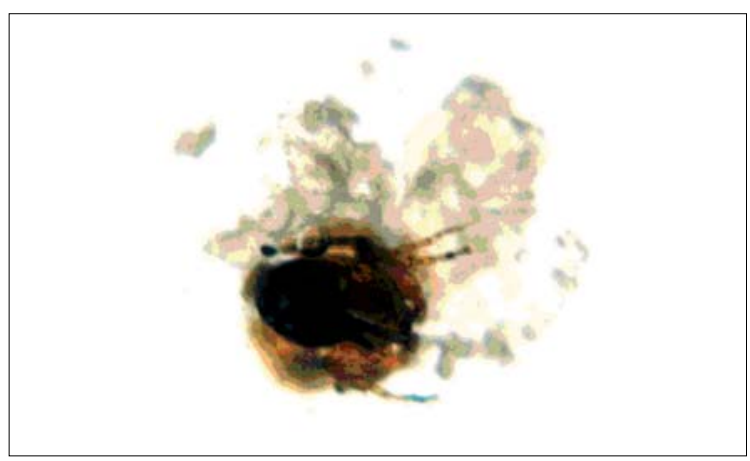

Figura 4. Material extraído de la lesión manipulada por el paciente (hembra de $T$. penetrans).

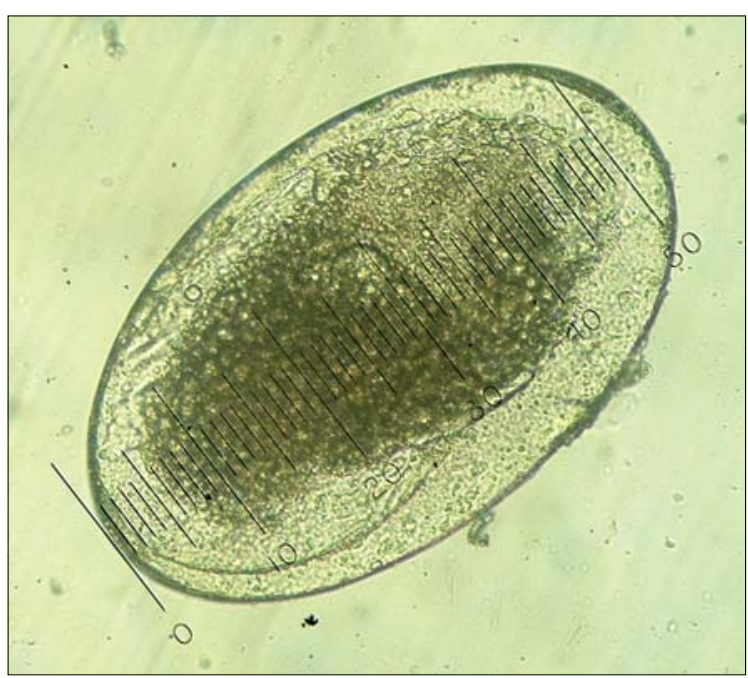

Figura 6. Visión microscópica de huevo de T. penetrans recientemente extraído (Aumento 40x). 


\section{Discusión}

Tunga penetrans fue descrita por primera vez en Haití 1525 por González Fernando de Oviedo y Valdés entre un grupo de conquistadores españoles. En 1623, Aleixo de Abreu realizó la primera descripción científica en Brasil. A comienzos del siglo XIX, la pulga se distribuyó hacia Angola y a fines del mismo siglo, a Madagascar. Posteriormente, se describió su presencia en India en el siglo $\mathrm{XX}^{2,3}$.

Tunga penetrans se caracteriza por ser la especie de pulga más pequeña, con $1 \mathrm{~mm}$ de longitud, de color marrón y tiene la capacidad de saltar hasta $35 \mathrm{~cm}$ del suelo $^{3}$. Su hábitat está constituido por suelo seco, arenoso, sombreado y templado, así como por suelos de cobertizos, viviendas, bodegas y establos de animales. Morfológicamente se caracteriza por presentar una cabeza triangular, oblicua hacia abajo y hacia delante, presenta pelos cortos en la frente, y un par de pequeños ojos negros. El rostro posee dos mandíbulas rudimentarias, un par de palpos tetra-articulados, un labio superior, un par de mandíbulas largas, anchas y dentadas y una epifaringe ${ }^{7}$. El tórax posee tres segmentos que se acortan en la parte anterior y el abdomen se subdivide en siete segmentos bien definidos, adquiriendo una forma puntiaguda en el macho y ovalada en la hembra ${ }^{8}$. Los apéndices locomotores tienen cinco segmentos y el tarso piloso termina en un par de uñas ${ }^{7}$.

Machos y hembras no fecundadas son hematófagas, siendo el ser humano un hospedero accidental. Luego de la cópula, el macho muere y la hembra grávida se fija por su rostro y penetra la piel, liberando kerato-enzimas. Tan pronto ingresa a la piel, la hembra comienza a producir, hasta 200 huevos, el abdomen crece alcanzando hasta $1 \mathrm{~cm}$ y la apertura genital de la hembra sobresale por el poro de la superficie cutánea del hospedero, a través del cual respira y elimina los huevos. Debido al gran tamaño que alcanza el abdomen, la pulga adquiere un aspecto piriforme o redondeado. Terminada la postura la hembra muere ${ }^{2,3}$.

El ciclo de vida de $T$. penetrans se desarrolla mediante metamorfosis holometábola y consta de huevo, dos estadíos larvales, pupa e imago. Los huevos eliminados sobre el terreno son ovalados y miden $600 \mu \times$ $320 \mu$ (Figura 6). Las larvas ápodas eclosionan, en promedio, al cabo de tres a cuatro días después de la postura; prefieren los lugares cálidos y secos como suelos arenosos de playas y zonas rurales $^{9-11}$, próximos a chiqueros, acúmulos de estiércol y en el peridomicilio $^{12-15}$. Se alimentan de materia vegetal y otros detritus ${ }^{16}$. La larva sufre una ecdisis que demora otro día (larva 2), luego empieza a pupar, entre cuatro y 10 días (en promedio 5-7 días). La formación de los imagos dentro del pupario ocurre dentro de nueve a 15 días (máxima eclosión en el día 12); sin embargo, pueden permanecer dentro del pupario hasta un año en ambientes fríos. En condiciones óptimas, la transición de huevo a adulto puede ser tan breve como 18 días, pero, en promedio, la metamorfosis completa dura un mes en condiciones favorables de temperatura y hu$\operatorname{medad}^{17}$.

Presenta una amplia gama de hospederos selváticos, domésticos y mascotas. Se la ha detectado infestando monos, ratas, ratones, cerdos, vacas, caballos, mulas, ovejas, cabras, perros, gatos, aves de corral y al hombre $^{18-22}$. Suele adquirirse la pulga al caminar descalzo en la tierra arenosa en regiones endémicas de la enfermedad.

La mayor prevalencia de tungiasis (31 a 42\%) ocurre en áreas rurales de Trinidad-Tobago, Norte de Brasil y Nigeria $^{2,3,6}$. Hay reportes de casos en E.U.A., Europa y Australia, los que han correspondido a viajeros que retornaron desde áreas endémicas ${ }^{23-26}$. En Chile, sólo se ha comunicado un caso en $1992^{27}$.

La clasificación de Fortaleza, propuesta en el año $2003^{28}$, describe la historia natural de la infestación humana por $T$. penetrans separándola en cinco estadios:

Estadio 1: Fase de penetración. Las hembras que han alcanzado la piel comienzan la penetración en el hospedero a los cinco minutos. La fase dura en total entre tres y siete horas, dependiendo del grosor de la piel. Habitualmente es asintomática pero puede ocasionar leve eritema de la piel.

Estadio 2: Hipertrofia del segmento abdominal. Comienza uno o dos días luego de la penetración. Se forma un halo eritematoso y pruriginoso con un diámetro hasta $30 \mathrm{~mm}$ alrededor de la lesión y un punto central pardo o negruzco constituido por la zona anogenital de la pulga.

Estadio 3: Halo blanco. Se inicia a las 72 horas post penetración. La hipertrofia es máxima y el abdomen adquiere forma esférica. Aparece un halo blanco alrededor del punto central, de consistencia firme, con forma similar a un vidrio de reloj, asociado a exudado amarillento parduzco y eliminación de material fecal. Los pacientes refieren sensación de cuerpo extraño, dolor pulsátil, eritema y calor local. En la fase de mayor desarrollo de este estadio, comienza la liberación de huevos, observándose a los seis días desecación y disminución de consistencia, manteniéndose el punto central pardo negruzco.

Estadio 4: Fase de involución. La pulga muere y deja de eliminar huevos. Se inicia en la tercera y finaliza en la quinta semana, hasta que se eliminan completa- 
mente los restos del artrópodo. La piel se aprecia arrugada y de color pardo negruzco.

Estadio 5: Formación de cicatriz en el estrato córneo. Todo el proceso dura entre cuatro y seis semanas.

La tungiasis se asocia a sobreinfección bacteriana ${ }^{29}$ por Streptococcus pyogenes, Streptococcus $\beta$ hemolítico no grupo A, Klebsiella aerogenes, Enterobacter agglomerans, Staphylococcus aureus, Escherichia coli y Bacillus $\mathrm{sp}^{10,30}$, principalmente en pacientes donde se manipuló la lesión con instrumentos no estériles $^{29}$. Asimismo, las lesiones pueden ser puerta de entrada de microorganismos como Clostridium tetani en pacientes sin inmunización ${ }^{31}, C$. perfringens y del hongo Paracoccidioides brasiliensis (blastomicosis).

Recientemente, se han identificado bacterias endosimbiontes del género Wolbachia en los ovarios de $T$. penetrans ${ }^{32}$, cuyos antígenos se han asociado con una respuesta immune patológica en enfermedades como la oncocercosis, por lo cual, parte de la respuesta immune en tungiasis podría atribuirse a antígenos de Wolbachia liberados con la muerte de las pulgas ${ }^{33}$.

En tungiasis puede haber complicaciones como la formación de úlceras, abscesos, flegmones, osteomielitis, linfangitis y gangrena de la piel. También se ha descrito la asociación con micosis profunda, neuritis ascendente, auto-amputación de ortejos y tétanos, en casos más graves ${ }^{2,34}$.

El diagnóstico se basa en las características clínicas de la lesión incluyendo el antecedente de viajes realizados a zonas endémicas de tungiasis, la morfología y localización de las lesiones. Se ha empleado la dermatoscopia como un método de diagnóstico eficaz para esta infestación ${ }^{35-36}$, en la cual se describe un anillo marrón central y un área de velo negro-azulado distribuidos en un patrón radial, que corresponden a los huevos ${ }^{37}$. No está indicada una biopsia de la lesión ni el examen histopatológico. Sin embargo, en Europa y América del Norte se efectúan cortes histológicos, como se efectuó en este caso, para confirmar el diagnóstico en viajeros que regresaban desde zonas endémicas $^{38}$. Los cortes demuestran la presencia del parásito, los huevos o fragmentos quitinosos ${ }^{38-41}$. En tungiasis de localización atípica, puede indicarse una biopsia, como sucede en las lesiones con aspecto pseudoepiteliomatoso en sitios ectópicos ${ }^{42}$.

El tratamiento de elección es la extirpación de las lesiones y de la pulga bajo condiciones estériles, tan pronto sea posible, para evitar la sobreinfección. Luego de la extracción pueden utilizarse antimicrobianos de uso tópico como mupirocina al $2 \%$ aplicándose dos veces al día por siete a 10 días. El toxoide tetánico debe ser administrado a todos los pacientes no vacunados o que recibieron su última dosis de vacuna anti-tetánica hace más de cinco años $2,3,34$.

Cuando las lesiones son muy numerosas o hay pústulas, puede ser necesario el empleo de antimicrobianos sistémicos ${ }^{34}$. En un trabajo clínico randomizado efectuado en 108 pacientes, se demostró que el uso tópico de ivermectina (loción), tiabendazol (ungüento y loción) o metrifonato (loción) versus un placebo (loción) redujo significativamente el número de lesiones. Sin embargo, estos fármacos no fueron eficaces para producir la lisis de la pulga en la fase de penetración ${ }^{31}$. También se ha utilizado ivermectina por vía oral, pero no se obtuvieron resultados significativos ${ }^{43,44}$.

Se ha recomendado a los viajeros a las zonas endémicas el uso de calzados cerrados, idealmente botas y evitar sentarse, o incluso tumbarse, en los sitios que habita esta pulga (medidas que son rechazadas por los turistas), evitar la cercanía de animales domésticos y realizarse autoexamen diario con el objeto de detectar lesiones incipientes, así como, el uso de repelentes durante todo el tiempo de permanencia en la $\mathrm{zona}^{34,44}$. Los repelentes registrados por EPA incluyen productos que contienen DEET (N,N-dietil-meta-toluamida) en concentraciones de 30 a $50 \%$ que son eficaces durante varias horas, se recomienda para adultos y niños a partir de los dos meses de vida. Icaridin (KBR 3023) disponible a concentraciones de 7 y $15 \%$ requiere aplicaciones más frecuentes. Se recomienda retirar el repelente al final del día antes de $\operatorname{acostarse}{ }^{45}$. Un repelente en base a aceite de coco, recomendado contra picaduras de insectos y garrapatas, ha sido eficaz en la regresión de la patología clínica de individuos gravemente infestados, como en la prevención de la reinfestación ${ }^{46,47}$.

En áreas no endémicas como Chile, esta ectoparasitosis constituye un problema diagnóstico. Debido al aumento de viajes internacionales a destinos tropicales, consideramos necesario informar tanto a los viajeros sobre las medidas de prevención de esta enfermedad como a los médicos sobre sus manifestaciones clínicas, conducta diagnóstica y terapéutica.

\section{Resumen}

La tungiasis es una ectoparasitosis cutánea producida por la hembra de la pulga de arena Tunga penetrans, cuya mayor prevalencia ocurre en África Sub-sahariana, Sudamérica y el Caribe. Comunicamos el caso de un chileno de 23 años que viajó a Brasil y que a su regreso presentó lesiones dérmicas sugerentes de tungiasis. El diagnóstico fue confirmado por una biopsia identificando el artrópodo y un huevo en una de las lesiones. Se revisa la historia natural, las co-morbilidades asociadas y alternativas de tratamiento. 


\section{Referencias}

1.- Brumpt E. Précis de Parasitologie II Cinquiéme Edition París France. Masson et Cie Editeurs 1936: 1335-8.

2.- Sachse M, Guldbakke K, Khachemoune A. Tunga penetrans: a stowaway from around the world. J Eur Acad Dermatol Venereol 2007; 21: 11-6.

3.- Heukelbach J. Tungiasis. Rev Inst Med Trop Sao Paulo 2005; 47: 307-13.

4.- Heukelbach J, Wilcke T, Eisele M. Ectopic localization of tungiasis. Am J Trop Med Hyg 2002; 67: 214-6.

5.- Kaimbo W, Bifuko A, van Ginderdeuren R. Upper eyelid localization of Tunga penetrans. Ophthalmologica 2007; 221: 439-42.

6.- Cestari T F, Pessato S, Ramos-e-Silva M. Tungiasis and myiasis. Clin Dermatol 2007; 25: $158-64$

7.- Núñez R. Tungiasis. Gac Med Méx 1952; 82: 11-5.

8.- Bastarrika G, Valcayo A, Vives R, Tunóón T, Santamaría M. Tungiasis: parasitosis viajera. An Sist Sanit Navar 1998; 21: 391-6.

9.- Heemskerk J, Empel I V, Jakimowicz J J. Tunga penetrans - A case report and review of the literature. Acta Chir Belg 2005; 105: 548-50.

10.- Heukelbach J, Oliveira F A S, Hesse G, Feldmeier H. Tungiasis: a neglected health problem of poor communities. Trop Med Int Health 2001; 6: 267-72.

11.- Bezerra S. Tungiasis - An unusual case of severe infestation. Int J Dermatol 1994; 33: 725 .

12.- Jelliffe D. Tungiasis. En: Simons RDG Ph (ed) - Handbook of Tropical Dermatology and Medical Mycology. 1953. Elsevier. Amsterdam, pp. 895-900.

13.- Neves D I D. Parasitología Humana. $4^{\mathrm{a}}$ Ed. 1979. Río de Janeiro. Ed. Guanabara Koogan S.A.

14.- Pessoa S B, Martins A, Pessoa V. Parasitología Médica, 10 a Ed. 1978, Río de Janeiro. Ed. Guanabara Koogan S.A.

15.- Rey L. Parasitología. Río de Janeiro, Ed. Guanabara Koogan S.A., 1972.

16.- Costa Lima A-Suctoria. En: Insetos do Brasil. Escola Nacional de Agronomia, 1943. Río de Janeiro. Imprensa Nacional 4: 17-71.

17.- Darmstadt G, Francis J. Tungiasis in a young child adopted from South America. Pediatr Infect Dis J 2000; 19: 485-7.

18.- Heukelbach J, da Costa A L, Wilcke T, Mencke N, Feldmeier H. The animal reservoir of Tunga penetrans in severely affected communities of north-east Brazil.
Med Vet Entomol 2004; 18: 329-35.

19.- Cooper J E. An outbreak of Tunga penetrans in a pig herd. Vet Rec 1967; 80: 365-6.

20.- Rietschel W. Observations of the sand flea (Tunga penetrans) in humans and dogs in French Guiana. Tierarztl Prax 1989; 17: 189-93.

21.- Trentini M, Pampiglione S, Giannetto S, Finocchiario B. Observations about specimens of Tunga sp (Siphonaptera, Tungidae) extracted from goats of Ecuador. Parassitología 2000; 42: 65.

22.- Sánchez-Maroto A, Peña L, Fernández R, Ladrón de Guevara C. Lesión dérmica en paciente con viaje reciente a Venezuela. Enferm Infecc Microbiol Clin 2002; 20: 221-2.

23.- Veraldi S, Valsecchi M. Imported tungiasis: a report of 19 cases and review of the literature. Int J Dermatol 2007; 46: 1061-6.

24.- Franck S, Feldmeier H, Heukelbach J. Tungiasis: more than an exotic nuisance. Travel Med Infect Dis 2003; 1: 159-66.

25.- Mashek H, Licznerski B, Pincus S. Tungiasis in New York. Int J Dermatol 1997; 36: 276-8.

26.- Spradbery J, Bromley J, Dixon R, Tetlow L. Tungiasis in Australia: an exotic disease threat. Med J Aust 1994; 161: 173.

27.- Spronhle V, Apt W, Eguiguren G, Pérez C, Zulantay I. Tungiasis. A clinical case. Rev Méd Chile 1992; 120: 794-6.

28.- Eisele M, Heukelbach J, Van Marck E, Mehlhorn H, Meckes O, Franck S, et al. Investigations on the biology, epidemiology, pathology and control of Tunga penetrans in Brazil: I. Natural history of tungiasis in man. Parasitol Res 2003; 90: 87-99.

29.- Feldmeier H, Heukelbach J, Eisele M, Sousa A Q, Barbosa L M, Carvalho C B. Bacterial superinfection in human tungiasis. Trop Med Int Health 2002; 7: 559-64.

30.- Chadee D D. Tungiasis among five communities in south-western Trinidad, West Indies. Ann Trop Med Parasitol 1998; 92: $107-13$.

31.- Heukelbach J, Eisele M, Jackson A, Felmeier H. Topical treatment of tungiasis: a randomized, controlled trial. Ann Trop Med Parasitol 2003; 97: 743-9.

32.- Fischer P, Schmetz C, Bandi C, Bonow I, Mand S, Fischer K, et al. Tunga penetrans: molecular identification of Wolbachia endobacteria and their recognition by antibodies against proteins of endobacteria from filarial parasites. Exp Parasitol 2002; 102: 201-11

33.- Saint Andre A, Blackwell N M, Hall L R,
Hoerauf A, Brattg N W, Volkmann L, et al. The role of endosymbiotic Wolbachia bacteria in the pathogenesis of river blindness. Science 2002; 295: 1892-5.

34.- Kehr J D, Heukelbach J, Mehlhorn H, Feldmeier H. Morbidity assessment in sand flea disease (tungiasis). Parasitol Res 2007; 100: 413-21.

35.- Bauer J, Forschner A, Garbe C, Röcken M. Dermoscopy of tungiasis. Arch Dermatol 2004; 140: 761-3.

36.- Bauer J, Forschner A, Garbe C, Röcken M. Variability dermoscopic features of tungiasis. Arch Dermatol 2005; 141: 643-4

37.- Di Stefani A, Rudolph C, Hofmann-Wellenhof R, Müllegger R. Dermoscopic features of tungiasis. Arch Dermatol 2005; 141: 1045-6.

38.- Franck S, Feldmeier H, Heukelbach J. Tungiasis: more than an exotic nuisance. Travel Med infect Dis 2003; 1: 159-66.

39.- Smith M D, Procop G W. Typical histologic features of Tunga penetrans in skin biopsies. Arch Path Lab Med 2002; 126: 714-16.

40.- Douglas-Jones A, Llewelyn M, Mills C. Cutaneous infection with Tunga penetrans. Br J Derm 1995; 133: 125-7.

41.- Macias P, Sashida P. Cutaneous infestation by Tunga penetrans. Int J Derm 2000; 39: 296-8.

42.- Smith M, Procop G. Typical histologic features of Tunga penetrans in skin biopsies. Arch Path Lab Med 2002; 126: 714-6.

43.- Heukelbach J, Sahebali S, van Marck E, Sabóia Moura R C, Feldmeier H. Pseudoepitheliomatous hyperplasia in ectopic tungiasis: an unusual case. Braz J Infect Dis 2004; 8: 465-8.

44.- Heukelbach J, Franck S, Feldmeier H. Therapy of tungiasis: a double-blinded randomized controlled trial with oral ivermectin. Mem Inst Oswaldo Cruz 2004; 99: 873-6.

45.- Heukelbach J. Review on tungiasis: treatment options and prevention. Expert Rev Anti Infect Ther 2006; 4: 151-7.

46.- No author listed. Measures to prevent bites from mosquitoes, ticks, fleas and other insects and arthropods. http://wwwn.cdc.gov/ travel/default.aspx. (accedido 6 octubre 2008).

47.- Feldmeier H, Kehr J D, Heukelbach J. A plant-based repellent protects against Tunga penetrans infestation and sand flea disease. Acta Tropica 2006; 99: 126-36

48.- Schwalfenberg S, Witt L H, Kehr J D, Feldmeier H, Heukelbach J. Prevention of tungiasis using a biological repellent: a small case series. Ann Trop Med Parasitol 2004; 98: 89-94. 